\title{
UNIVALENT FUNCTIONS AND THE POMPEIU PROBLEM
}

\author{
NICOLA GAROFALO AND FAUSTO SEGALA
}

\begin{abstract}
In this paper we prove a result on the Pompeiu problem. If the Schwarz function $\Phi$ of the boundary of a simply-connected domain $\Omega \subset \mathbb{R}^{2}$ extends meromorphically into a certain portion $E$ of $\Omega$ with a pole at some point $z_{0} \in E$, then $\Omega$ has the Pompeiu property unless $\Phi$ is a Möbius transformation, in which case $\Omega$ is a disk.
\end{abstract}

\section{INTRODUCTION}

In 1929 the Rumanian mathematician D. Pompeiu formulated the following problem: "To characterize those bounded domains $\Omega \subset \mathbb{R}^{2}$ for which $f \equiv 0$ is the only continuous function such that

$$
\int_{\sigma(D)} f d x=0
$$

for every rigid motion $\sigma$ of $\mathbb{R}^{2}$.

One says that $\Omega$ has the Pompeiu property if $f \equiv 0$ is the only continuous function for which (1.1) holds. For a historical introduction to the problem we refer the reader to [GS1]. In that paper we conjectured that (modulo sets of measure zero) the disk is the only simply-connected domain that does not have the Pompeiu property. Chakalov [C] was the first one to realize that the disk fails to have the Pompeiu property. In fact, if one considers the function $f\left(x_{1}, x_{2}\right)=\sin \left(a x_{1}\right)$, then one has

$$
\int_{B_{r}\left(x_{0}\right)} f(x) d x=\frac{2 \pi r}{a} \sin \left(a x_{0,1}\right) J_{1}(a r),
$$

where $x_{0}=\left(x_{0,1}, x_{0,2}\right)$ is fixed, $B_{r}\left(x_{0}\right)=\left\{x|| x-x_{0} \mid<r\right\}$, and $J_{1}$ is the Bessel function of order one. It is therefore enough to choose $a>0$, such that $J_{1}(a r)=0$, for (1.1) to hold.

This paper contains some progress toward the above conjecture. Let $\Omega \subset \mathbb{R}^{2}$ be a bounded simply-connected domain whose boundary $\partial \Omega$ is a piecewise $C^{1}$ Jordan curve. By the Riemann mapping theorem there exists a univalent function $h: D \rightarrow \Omega$, where $D=\{z \in \mathbb{C}|| z \mid<1\}$. Moreover, $h$ can be extended in a one-to-one fashion to a continuous map of $\bar{D}$ onto $\bar{\Omega}$. In order

Received by the editors May 7, 1993.

1991 Mathematics Subject Classification. Primary 30E15, 41A60, 42 A38.

The first author was supported by the NSF grant DMS \#9104023. 
to state the main result in this paper we need to introduce some definitions. We consider the Schwarz function of $\partial \Omega$ given by

$$
\Phi(w)=\overline{h\left(\frac{1}{\overline{h^{-1}(w)}}\right)} .
$$

A priori, $\Phi$ is well defined on $\partial \Omega$. Given a straight line $L \subset \mathbb{C}$, and a point $z_{0} \notin L$, we denote by $\Lambda\left(L ; z_{0}\right)$ the open half-plane lying on one side of $L$ and containing $z_{0}$. We also let

$$
E\left(L ; z_{0}\right)=\Lambda\left(L ; z_{0}\right) \cap \Omega .
$$

The main result in this paper is given by the following

Theorem 1. Suppose that there exist $z_{0} \in \Omega$ and a straight line $L \subset \mathbb{C}$ such that:

(i) $\Phi$ can be extended to a holomorphic function in $E\left(L ; z_{0}\right) \backslash\left\{z_{0}\right\}$ having a pole in $z_{0}$;

(ii) $\Phi$ is not a Möbius transformation.

Then, $\Omega$ has the Pompeiu property.

Figure 1 below illustrates the situation.

We now state two remarkable consequences of Theorem 1.

Corollary 2. Suppose that $h$ is univalent in $D$ and meromorphic in $\mathbb{C}$, with at least one pole in $\overline{\mathbb{C}} \backslash \bar{D}$. If, moreover, $h$ is not a Möbius transformation, then $\Omega=h(D)$ has the Pompeiu property.

If we specialize Theorem 1 to the class of convex domains we obtain the following partial solution of the Pompeiu problem.

Corollary 3. Suppose that $\Omega=h(D)$ be a convex set. Assume that $h$ has a pole on the boundary of the circle of convergence relative to its Taylor expansion at $z=0$. If $h$ is not a Möbius transformation, then $\Omega$ has the Pompeiu property.

Remark. Corollary 2 contains the result in our paper [GS2] (see also [GS3]) concerned with the case

$$
h(z)=\sum_{k=0}^{N} a_{k} z^{k} .
$$

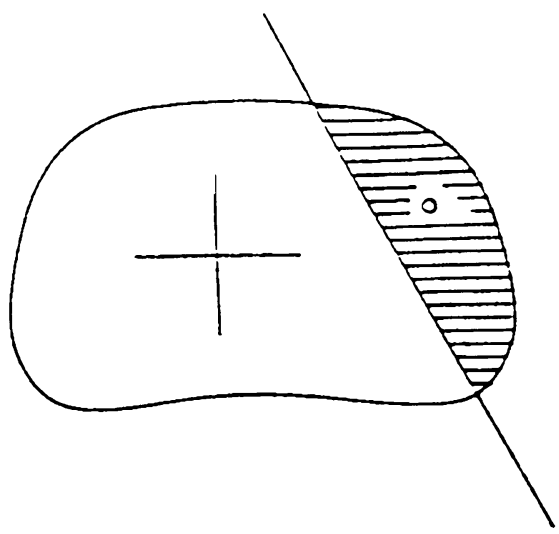

Figure 1 
Furthermore, it contains a result in a recent paper by Ebenfelt [E]. The latter has proved that if $h$ is a univalent function in $D$ such that $h(z)=\frac{p(z)}{q(z)}$, with $p$ and $q$ polynomials, then $\Omega=h(D)$ has the Pompeiu property, unless $h$ is a Möbius transformation.

Our strategy to prove Theorem 1 is to study, by Riemann's method of the steepest descent, the asymptotic behavior of the (complexified) Fourier transform of the characteristic function of $\Omega, \hat{\chi}_{\Omega}$, along the algebraic variety of $\mathbb{C}^{2}$, $M_{\alpha}=\left\{\zeta_{1}^{2}+\zeta_{2}^{2}=\alpha\right\}, \alpha>0$. This is due to an important characterization of the Pompeiu property established in 1973 by Brown, Schreiber, and Taylor [BST], see Theorem $A$ in the next section. We mention that Berenstein $[B]$ was the first one to use asymptotic expansions of $\hat{\chi}_{\Omega}$ in connection with the Pompeiu problem.

\section{Preliminary Reductions}

We begin this section by recalling the above-mentioned characterization of the Pompeiu property due to Brown, Schreiber, and Taylor [BST].

Theorem A. A bounded domain $\Omega \subset \mathbb{R}^{2}$ has the Pompeiu property if and only if there exists no $\alpha \in \mathbb{C} \backslash\{0\}$ such that the complexified Fourier transform of the characteristic function of $\Omega, \hat{\chi}_{\Omega}$, vanishes identically on

$$
M_{\alpha}=\left\{\left(\zeta_{1}, \zeta_{2}\right) \in \mathbb{C}^{2} \mid \zeta_{1}^{2}+\zeta_{2}^{2}=\alpha\right\}
$$

It was observed by Berenstein [B] that, when $\Omega$ is simply connected, $\alpha \in$ $\mathbb{C} \backslash\{0\}$ in the statement of Theorem A can be replaced by $\alpha>0$. Furthermore, when $\partial \Omega$ is a rectifiable Jordan curve, then the divergence theorem allows to replace $\hat{\chi}_{\Omega}$ with $\hat{\chi}_{\partial \Omega}$. Note that for $\zeta=\left(\zeta_{1}, \zeta_{2}\right) \in \mathbb{C}^{2}$

$$
\hat{\chi}_{\partial \Omega}=\int_{\partial \Omega} e^{i\langle\zeta, x\rangle}\left(d x_{1}+i d x_{2}\right)
$$

where we have let $\langle\zeta, x\rangle=\zeta_{1} x_{1}+\zeta_{2} x_{2}$. Changing $\zeta$ in $-i \zeta$ in (2.1) we are thus led to study the following oscillatory integral

$$
\int_{\partial \Omega} e^{\langle\zeta, x\rangle}\left(d x_{1}+i d x_{2}\right)
$$

for $\zeta \in M_{-\alpha}$, with $\alpha>0$. We write $\zeta$ in the form

$$
\zeta=r(\cos \theta, \sin \theta)+i t(-\sin \theta, \cos \theta) .
$$

The condition $\zeta \in M_{-\alpha}$ becomes

$$
t^{2}=\alpha+r^{2}
$$

We have

$$
\begin{aligned}
\langle\zeta, x\rangle & =x_{1}(r \cos \theta-i t \sin \theta)+x_{2}(r \sin \theta+i t \cos \theta) \\
& =r x_{1} e^{-i \theta}+i r x_{2} e^{-i \theta}-i(t-r) x_{1} \sin \theta+i(t-r) x_{2} \cos \theta \\
& =r e^{-i \theta}\left(x_{1}+i x_{2}\right)-i(t-r)\left(x_{1} \sin \theta-x_{2} \cos \theta\right) .
\end{aligned}
$$

Since from our assumptions in the introduction $\partial \Omega=h(\partial D)$, where $h$ is univalent in $D=\{w \in \mathbb{C}|| w \mid<1\}$, we have for $s \in[0,2 \pi]$

$$
x_{1}(s)=\frac{1}{2} h\left(e^{i s}\right)+\frac{1}{2} k\left(e^{i s}\right) .
$$



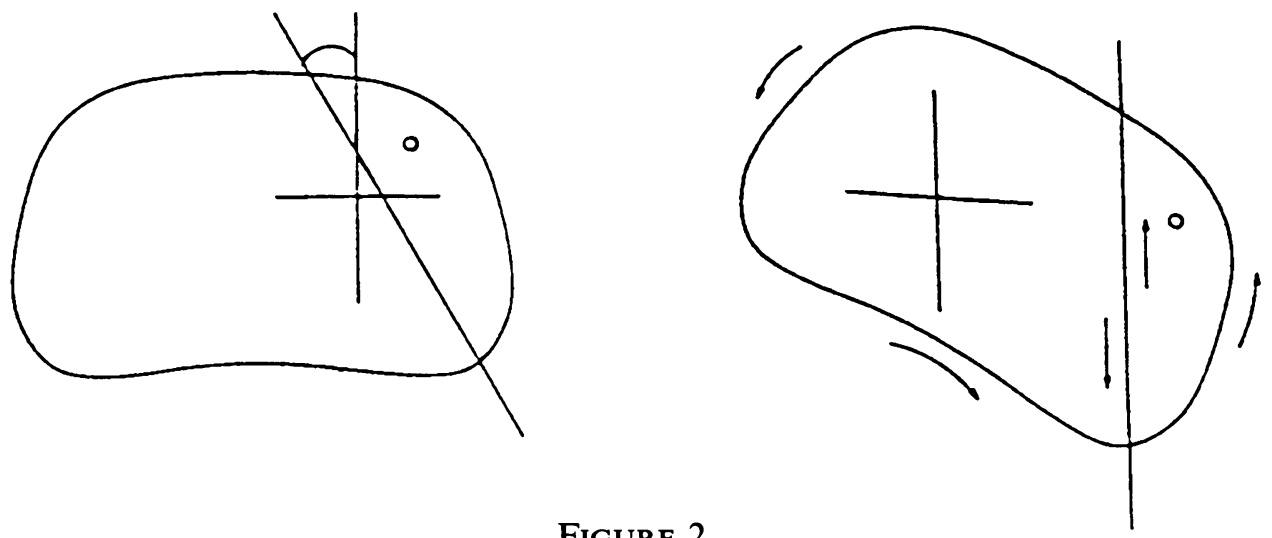

FIGURE 2

Here, we have let

$$
k(w)=\overline{h\left(\frac{1}{\bar{w}}\right)} .
$$

Analogously, we have

$$
x_{2}(s)=\frac{1}{2 i} h\left(e^{i s}\right)-\frac{1}{2 i} k\left(e^{i s}\right) .
$$

Inserting (2.5), (2.7) in (2.4) we obtain

$$
\langle\zeta, x\rangle=r e^{-i \theta} h-i \frac{(t-r)}{2}[(h+k) \sin \theta+i(h-k) \cos \theta],
$$

which, after some easy reductions, gives

$$
\langle\zeta, x\rangle=\frac{t+r}{2} e^{-i \theta} h\left(e^{i s}\right)-\frac{t-r}{2} e^{i \theta} k\left(e^{i s}\right) .
$$

Taking (2.8) into account, we see that (up to a factor of $i$ ) the integral in (2.2) becomes

$$
\begin{aligned}
I(r) & =\int_{\partial \Omega} \exp \left[\frac{t+r}{2} e^{-i \theta} w-\frac{t-r}{2} e^{i \theta} \Phi(w)\right] d w \\
& =e^{i \theta} \int_{\partial \Sigma} \exp \left[\frac{t+r}{2} w-\frac{t-r}{2} \Psi(w)\right] d w
\end{aligned}
$$

where $\Sigma=e^{-i \theta} \Omega, \Psi(w)=e^{i \theta} \Phi\left(e^{i \theta} w\right)$. At this point we choose $\theta \in[0,2 \pi]$ in such a way that the straight line $e^{-i \theta} L$, where $L$ is as in the statement of Theorem 1, becomes parallel to the imaginary axis. We let $w_{0}=e^{-i \theta} z_{0}, M=$ $e^{-i \theta} L$, where $z_{0} \in \Omega$ is as in the assumption of Theorem 1, see Figure 2.

We now have from (2.9)

$$
e^{-i \theta} I(r)=\left(\int_{\partial E\left(M ; w_{0}\right)}+\int_{\partial\left[\Sigma \backslash E\left(M ; w_{0}\right)\right]}\right) \exp \left[\left(\frac{t+r}{2}\right) w-\frac{t-r}{2} \Psi(w)\right] d w .
$$

\section{Asymptotic EXPANSION OF $\hat{\chi}_{\partial \Omega}$ AND the PoMPEIU PROPERTY}

The aim of this section is to establish the asymptotic behavior as $r \rightarrow \infty$ of the integral in the right-hand side of (2.10). We begin by analyzing that 
part of the integral on the set $\partial\left(\Sigma \backslash E\left(M ; w_{0}\right)\right)$. We let $A=\max |\Psi|$ on $\partial\left(\Sigma \backslash E\left(M ; w_{0}\right)\right)$. Then

$$
\begin{aligned}
\mid \int_{\partial\left(\Sigma \backslash E\left(M ; w_{0}\right)\right)} & \exp \left[\frac{t+r}{2} w-\frac{t-r}{2} \Psi(w)\right] d w \mid \\
& \leq \int_{\partial\left(\Sigma \backslash E\left(M ; w_{0}\right)\right)} \exp \left[\frac{t+r}{2} \Re e w+\frac{t-r}{2} A\right] d s .
\end{aligned}
$$

We now choose $\beta>0$ such that on the set $\partial\left(\Sigma \backslash E\left(M ; w_{0}\right)\right)$ we have (see Figure 2)

$$
\mathfrak{R} e w \leq \mathfrak{R} e w_{0}-\beta .
$$

Noting that (2.3) gives

$$
\frac{t+r}{2}=r\left(1+O\left(\frac{1}{r^{2}}\right)\right), \quad t-r=\frac{\alpha}{2 r}\left(1+O\left(\frac{1}{r}\right)\right)
$$

as $r \rightarrow \infty$, it follows that on the set $\partial\left(\Sigma \backslash E\left(M ; w_{0}\right)\right)$ we have uniformly as $r \rightarrow \infty$

$\frac{t+r}{2} \mathfrak{R} e w+\frac{t-r}{2} A \leq \frac{t+r}{2}\left(\mathfrak{R} e w_{0}-\beta\right)+\frac{t-r}{2} A=r\left(\mathfrak{R} e w_{0}-\beta\right)(1+o(1))$.

From this we derive the estimate for $r \rightarrow \infty$

$$
\left|\int_{\partial\left(\Sigma \backslash E\left(M ; w_{0}\right)\right)} \exp \left[\frac{t+r}{2} w-\frac{t-r}{2}(w)\right] d w\right| \leq C \exp \left[r\left(\mathfrak{R} e w_{0}-\frac{\beta}{2}\right)\right] .
$$

We will now analyze the first integral in the right-hand side of (2.10). We have for $\delta>0$ small by Cauchy's theorem

$$
\begin{aligned}
\int_{\partial E\left(M ; w_{0}\right)} & \exp \left[\frac{t+r}{2} w-\frac{t-r}{2} \Psi(w)\right] d w \\
& =\exp \left(\frac{t+r}{2} w_{0}\right) \int_{\left|w-w_{0}\right|=\delta} \exp \left[\frac{t+r}{2}\left(w-w_{0}\right)-\frac{t-r}{2} \Psi(w)\right] d w \\
& =\exp \left(\frac{t+r}{2} w_{0}\right) \int_{|w|=\delta} \exp \left[\frac{t+r}{2} w-\frac{t-r}{2} \Psi\left(w_{0}+w\right)\right] d w .
\end{aligned}
$$

By the assumptions on $\Phi$ in Theorem 1, there exists $m \in \mathbb{N}$ such that

$$
\Psi\left(w_{0}+w\right)=\sum_{k=-m}^{\infty} a_{k} w^{k}
$$

for $|w| \leq \delta$, with $a_{-m} \neq 0$. We now distinguish two cases.

First case. $m \geq 2$.

Using (2.3) we can write

$$
\frac{t-r}{2}=\frac{\alpha}{2(t+r)} .
$$

By (3.4), (3.5) we have on the circle $\left\{w \in \delta e^{i \tau} \mid-\pi \leq \tau \leq \pi\right\}$

$$
\begin{aligned}
& \frac{t+r}{2} w-\frac{t-r}{2} \Psi\left(w_{0}+w\right) \\
& \quad=\frac{t+r}{2} \delta e^{i \tau}-\frac{\alpha a_{-m}}{2(t+r)} \delta^{-m} e^{-i m \tau}-\frac{\alpha}{2(t+r)} \sum_{k=-m+1} a_{k} \delta^{k} e^{i k \tau} .
\end{aligned}
$$


We now choose

$$
\delta=\left(\frac{t+r}{2}\right)^{-2 /(m+1)} .
$$

Then, (3.6) becomes as $r \rightarrow \infty$

$$
\frac{t+r}{2} w-\frac{t-r}{2} \Psi\left(w_{0}+w\right)=\left(\frac{t+r}{2}\right)^{(m-1) /(m+1)}\left[e^{i \tau}-\frac{\alpha a_{-m}}{4} e^{-i m \tau}+o(1)\right] .
$$

From the first equality in (3.1) we conclude that

$$
\frac{t+r}{2} w-\frac{t-r}{2} \Psi\left(w_{0}+w\right)=r^{(m-1) /(m+1)} q(r)\left\{e^{i \tau}-\frac{\alpha a_{-m} e^{-i m \tau}}{4}+o(1)\right\}
$$

with $q(r) \rightarrow 1$ as $r \rightarrow \infty$, uniformly on the circle $\left\{w=\delta e^{i \tau} \mid-\pi \leq \tau \leq \pi\right\}$. Taking (3.7) into account, we obtain for (3.3) with some $p(r) \rightarrow 1$ as $r \rightarrow \infty$ (3.8)

$$
\begin{gathered}
\int_{\partial E\left(M ; w_{0}\right)} \exp \left[\frac{t+r}{2} w-\frac{t-r}{2} \Psi(w)\right] d w=i r^{-2 /(m+1)} p(r) \exp \left(\frac{t+r}{2} w_{0}\right) \\
\cdot \int_{-\pi}^{\pi} \exp \left\{r^{(m-1) /(m+1)} q(r)\left[e^{i \tau}-\frac{\alpha a_{-m}}{4} e^{-i m \tau}+o(1)\right]\right\} e^{i \tau} d \tau .
\end{gathered}
$$

At this point we observe that the integral on the right-hand side of (3.8) is of the type studied in the paper [GS2]. By virtue of the work done in [GS2] we can conclude that the asymptotic behavior, as $r \rightarrow \infty$, of the above-mentioned integral is as follows

$$
\begin{aligned}
\int_{-\pi}^{\pi} & \exp \left\{r^{(m-1) /(m+1)} q(r)\left[e^{i \tau}-\frac{\alpha a_{-m}}{4} e^{-i m \tau}+o(1)\right]\right\} e^{i \tau} d \tau \\
& =r^{-(m-1) / 2(m+1)} A(r) \exp \left[r^{(m-1) /(m+1)} B(r)\right],
\end{aligned}
$$

where, having let $\varphi(\tau)=e^{i \tau}-\frac{\alpha a_{-m}}{4} e^{-i m \tau}$ for $\tau \in \mathbb{C}$, one has for $r \rightarrow \infty$

$$
A(r) \rightarrow \frac{e^{i \tau_{0}}}{\sqrt{2 \varphi^{\prime \prime}\left(\tau_{0}\right)}}=A_{0} \neq 0, \quad B(r) \rightarrow \varphi\left(\tau_{0}\right) .
$$

Here, $\tau_{0}$ is a suitable simple critical point of the function $\varphi$. Inserting (3.9) in (3.8) and recalling (3.1), we obtain

$$
\begin{aligned}
\int_{\partial E\left(M ; w_{0}\right)} & \exp \left[\frac{t+r}{2} w-\frac{t-r}{2} \Psi(w)\right] d w \\
& =r^{-(m+3) / 2(m+1)} A_{1}(r) \exp \left[r w_{0}+r^{(m-1) /(m+1)} B(r)\right],
\end{aligned}
$$

where $A_{1}(r) \rightarrow i A_{0}$, as $r \rightarrow \infty$.

Using (3.2), (3.10) in (2.10) we finally conclude for $r \rightarrow \infty$

$$
\begin{aligned}
e^{-i \theta} I(r)= & r^{-(m+3) / 2(m+1)} A_{1}(r) \exp \left[r w_{0}+r^{(m-1) /(m+1)} B(r)\right] \\
& \cdot\left\{1+O\left(r^{(m+3) / 2(m+1)} \exp \left[-\frac{\beta}{2} r+C r^{(m-1) /(m+1)}\right]\right)\right\},
\end{aligned}
$$

for some number $C>0$. Observing now that $0<\frac{m-1}{m+1}<1$, we infer that

$$
O\left(r^{(m+3) / 2(m+1)} \exp \left[-\frac{\beta}{2} r+C r^{(m-1) /(m+1)}\right]\right)=o(1)
$$


as $r \rightarrow \infty$. In conclusion, we obtain from (3.11)

$$
e^{-i \theta} I(r)=r^{-(m+3) / 2(m+1)} A_{2}(r) \exp \left[r w_{0}+r^{(m-1) /(m+1)} B(r)\right],
$$

where $A_{2}(r) \rightarrow i A_{0}$ as $r \rightarrow \infty$.

To conclude the study of the asymptotic behavior of the integral $e^{-i \theta} I(r)$ in (2.10) we need to analyze the case in which $\Psi$ has as simple pole in $w_{0}$, i.e., the case in which $m=1$ in (3.4).

Second case. $m=1$.

We consider again the integral on the circle $\left\{w \in \delta e^{i \tau} \mid-\pi \leq \tau \leq \pi\right\}$ in the right-hand side of (3.3). By the assumptions in Theorem 1, the function $\Psi$ is not a Möbius transformation. If $w=\sigma e^{i \tau}$, with $|\sigma|=\delta$, then we have from (3.5)

$$
\begin{aligned}
\frac{t+r}{2} w & -\frac{t-r}{2} \Psi\left(w_{0}+w\right)=\frac{1}{2}\left[(t+r) w-\frac{\alpha}{t+r} \Psi\left(w_{0}+w\right)\right] \\
& =\frac{1}{2}\left\{(t+r) \sigma e^{i \tau}-\frac{\alpha}{t+r}\left[\frac{a_{-1}}{\sigma} e^{-i \tau}+a_{0}+\sum_{k=q}^{\infty} a_{k} \sigma^{k} e^{i k \tau}\right]\right\}
\end{aligned}
$$

for some $q \in \mathbb{N}$, with $a_{q} \neq 0$. At this point we choose

$$
\sigma=\frac{i \sqrt{\alpha a_{-1}}}{t+r} \text {. }
$$

It follows from (3.13), (3.14) that for $w=\sigma e^{i \tau}$ one has

$$
\begin{gathered}
\frac{t+r}{2} w-\frac{t-r}{2} \Psi\left(w_{0}+w\right)=-\frac{\alpha a_{0}}{t+r}+i \sqrt{\alpha a_{-1}} \cos \tau \\
+\frac{C}{(t+r)^{q+1}} e^{i q \tau}+O\left(\frac{1}{(t+r)^{q+2}}\right),
\end{gathered}
$$

for some $C \neq 0$. We conclude

$$
\begin{aligned}
& \int_{|w|=\delta} \exp \left[\frac{t+r}{2} w-\frac{t-r}{2} \Psi\left(w_{0}+w\right)\right] d w \\
& \quad=i \sigma \varepsilon^{-\alpha a_{0} /(t+r)} \int_{-\pi}^{\pi} e^{i \sqrt{\alpha a_{-1}} \cos \tau}\left\{1+\frac{C}{(t+r)^{q+1}} e^{i q \tau}+O\left(\frac{1}{(t+r)^{q+2}}\right)\right\} e^{i \tau} d \tau .
\end{aligned}
$$

We now recall the integral representation of the Bessel function $J_{n}$ (see [L])

$$
J_{n}(z)=\frac{i^{-n}}{2 \pi} \int_{-\pi}^{\pi} e^{i z \cos \tau} e^{i n \tau} d \tau, \quad n \in \mathbb{Z} .
$$

Using this we can rewrite (3.16) as follows

$$
\begin{aligned}
& \int_{|w|=\delta} \exp \left[\frac{t+r}{2} w-\frac{t-r}{2} \Psi\left(w_{0}+w\right)\right] d w \\
& =-\frac{\sqrt{\alpha a_{-1}}}{t+r} e^{-\alpha a_{0} /(t+r)}\left\{2 \pi i J_{1}\left(\sqrt{\alpha a_{-1}}\right)\right. \\
& \left.+\frac{2 \pi C i^{q} J_{q}\left(\sqrt{\alpha a_{-1}}\right)}{(t+r)^{q+1}}+O\left(\frac{1}{(t+r)^{q+2}}\right)\right\} .
\end{aligned}
$$


Since, from (3.1), $t+r(1+o(1))$ as $r \rightarrow \infty$, and by a theorem of Siegel, $J_{1}$ and $J_{q}$ have no common zeros (see [W, p. 485]). (3.17) implies

$$
\begin{aligned}
\int_{|w|=\delta} & \exp \left[\frac{t+r}{2} w-\frac{t-r}{2} \Psi\left(w_{0}+w\right)\right] d w \\
& =\frac{E_{1}(r)}{r}\left[J_{1}\left(\sqrt{\alpha a_{-1}}\right)+\frac{E_{2}}{r^{q+1}} J_{q}\left(\sqrt{\alpha a_{-1}}\right)\right],
\end{aligned}
$$

where $E_{1}(r) \rightarrow E_{0} \neq 0$ as $r \rightarrow \infty$, and $E_{2} \neq 0$. From (2.10), (3.2) and (3.18) we finally obtain

$$
\varepsilon^{-i \theta} I(r)=\varepsilon^{r w_{0}} \frac{E_{3}(r)}{r}\left[J_{1}\left(\sqrt{\alpha q_{-1}}\right)+\frac{E_{2}}{r^{q+1}} J_{q}\left(\sqrt{\alpha a_{-1}}\right)\right]
$$

with $E_{3}(r) \rightarrow E_{0}$ as $r \rightarrow \infty$ (of course, in this estimate we have used again (3.1)).

We are now ready to conclude the proof of Theorem 1 . We recall that from the reductions in $\S 2$, the oscillatory integral $\hat{\chi}_{\partial \Omega}(\zeta)$, with $\zeta$ moving out to infinity along a special path of $M_{-\alpha}$, was shown to equal $e^{-i \theta} I(r)$ in (2.10) (up to a factor of $i$ ).

From (3.12), (3.19) we see that, under the assumptions in Theorem 1, the latter cannot vanish identically on $M_{-\alpha}$. From Theorem A we conclude that $\Omega$ has the Pompeiu property.

\section{Proofs of Corollaries 2 and 3}

The proof of Corollary 2 follows immediately from Theorem 1 by observing that if $\Phi$ is a Möbius transformation, then so is $h$. Moreover, if $h$ has at least one pole, then $\Phi$ has at least one pole and at most one essential singularity $(w=0)$.

As for the proof of Corollary 3 we observe that if $\Omega=h(D)$ is convex, then by Study's theorem [S, Theorem 2.4] so is $h\left(D_{r}\right)$ for $0<r \leq 1$, where $D_{r}=\{z \in \mathbb{C}|| z \mid \leq r\}$. Set $S=\{x \in \mathbb{C}|| z \mid<R\}$ with $R>1$, and denote $S^{-1}=\left\{\frac{1}{z} \mid z \in S\right\}$. Assume that $h$ is holomorphic in $S$ with a pole $z_{0}$ on $\partial S$. Then $\Phi$ is holomorphic on $h\left(S^{-1}\right)$ and has a pole in $h\left(\frac{1}{z_{0}}\right)$. Since $h\left(S^{-1}\right)$ is convex we are in a position to apply Theorem 1, see Figure 3.

We close this paper with an example of a one-parameter family of domains which fall within the scope of Theorem 1 , but are not included in any previous result on the Pompeiu problem.

Example. Consider for $0<\lambda<2$ the map $h_{\lambda}: D \rightarrow \mathbb{C}$ given by $h_{\lambda}(x)=\frac{e^{\lambda}}{\lambda_{z}-2}$. In Figure 4 , we represent $\Omega_{\lambda}=h_{\lambda}(D)$ for some values of $\lambda$. There exists $\lambda_{0} \in(0,2)$ such that for $0<\lambda<\lambda_{0}$ the domain $\Omega_{\lambda}$ is convex. Furthermore, one verifies that for $\lambda \in\left(0, \lambda_{0}\right)$

$$
\min \operatorname{diam} \Omega_{\lambda}>\frac{1}{2} \max \operatorname{diam} \Omega_{\lambda}
$$

so that the result in $[\mathrm{BK}]$ cannot be invoked. 

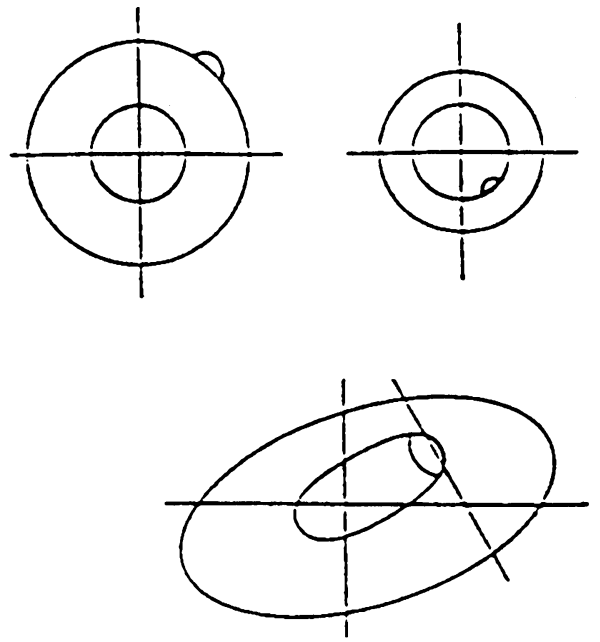

FIGURE 3

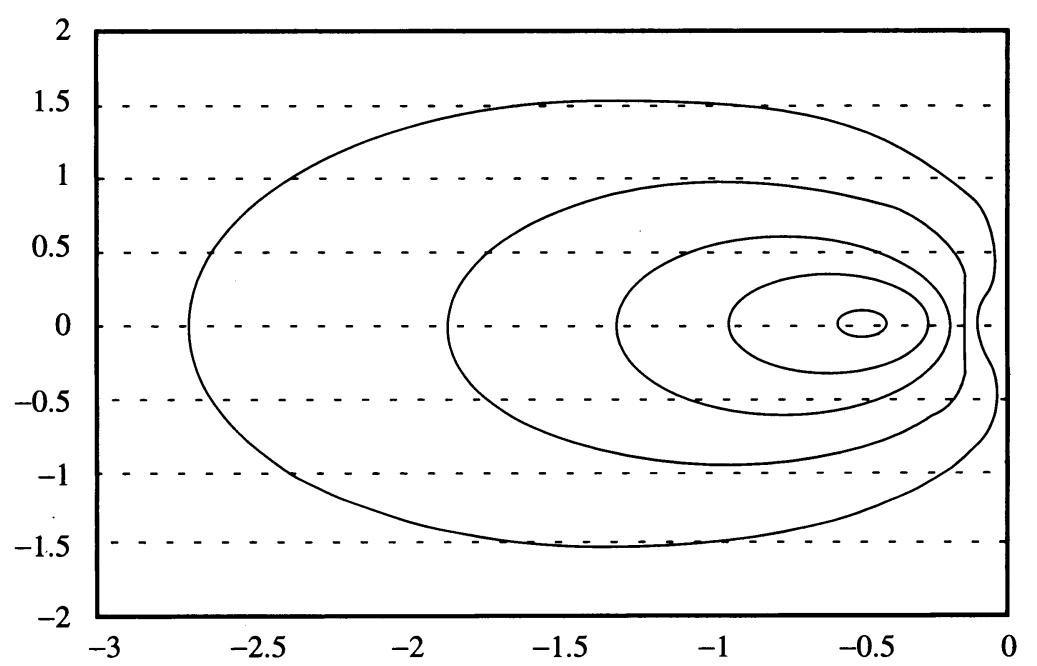

FIGURE 4

\section{ACKNOWLEDGMENT}

We would like to thank Tommaso Ruggeri and Yuting Wei for graciously helping us with the drawings.

\section{REFERENCES}

[B] C. A. Berenstein, An inverse spectral theorem and its relation to the Pompeiu problem, J. Analyse Math. 37 (1980), 124-144.

[BK] L. Brown and J. P. Kahane, A note on the Pompeiu problem for convex domains, Math. Ann. 259 (1982), 107-110. 
[BST] L. Brown, B. M. Schreiber, and A. B. Taylor, Spectral synthesis and the Pompeiu problem, Ann. Inst. Fourier (Grenoble) 23 (1973), 125-154.

[C] L. Chakalov, Sur un problème de D. Pompeiu, Annuaire Univ. Sofia Fac. Phys. Math. 40 (1944), 1-44.

[E1] P. Ebenfelt, Some results on the Pompeiu problem, preprint, 1992.

[E2] _ Propagation of singularities from singular and infinite points in certain complexanalytic Cauchy problems and an application to the Pompeiu problem, preprint, 1993.

[GS1] N. Garofalo and F. Segala, Asymptotic expansions for a class of Fourier integrals and applications to the Pompeiu problem, J. Analyse Math. 56 (1991), 1-28.

[GS2] _ New results on the Pompeiu problem, Trans. Amer. Math. Soc. 325 (1991), 273-286.

[GS3] _ Another step toward the solution of the Pompeiu in the plane, Comm. Partial Differential Equations (to appear).

[L] N. N. Lebedev, Special functions and their applications, Dover, New York, 1972.

[S] G. Schober, Univalent functions-Selected topics, Lecture Notes in Math., vol. 478, SpringerVerlag, Berlin and New York, 1975.

[W] G. N. Watson, A treatise on the theory of Bessel functions, 2nd ed., Cambridge Univ. Press, 1962.

[Z] L. Zalcman, A bibliographical survey of the Pompeiu problem, Approximation by Solutions of Partial Differential Equations, Quadrature Formulae and Related Topics, (M. Goldstein and W. Haussman, eds.), Kluwer, 1992.

Department, of Mathematics, Purdue University, West lafayette, Indiana 47907

Dipartimento di Matematica, Via Machiavelli 35, Università di Ferrara, 44100 FerRARA, ITALY 